\title{
JULIO RAMVÓN RIBEYRO Y LOS DEBATES LITERARIOS DE LOS AÑOS 60 EN EL PERÚ
}

Pedro Carlos Espinoza Huaroto*

pedrocjespinoza@gmail.com

Universidad Nacional Federico Villarreal

Fecha de recepción: agosto de 2019

Fecha de aceptación: diciembre de 2019

Resumen: El objetivo de nuestra investigación es evidenciar que los argumentos desarrollados por Julio Ramón Ribeyro en torno a la novela, la representación de la realidad y técnicas literarias expuestos en los artículos y ensayos de La caza sutil (1976) coinciden con los críticos literarios de su contexto. Estos manifiestan sus argumentos en los eventos literarios que se suscitaron en 1965: la

* Pedro Carlos Espinoza Huaroto es bachiller en Literatura por la Universidad Nacional Federico Villarreal (UNFV). Ha participado en el Congreso Internacional "Julio Ramón Ribeyro: por tiempo indefinido" (2014), el XVIII y XIX Coloquio de Estudiantes de Literatura PUCP y VII Congreso Internacional de Estudiantes de Literatura (CONELIT). Actualmente se desempeńa como docente y cursa la maestría de Literatura Infantil y Juvenil - Animación a la Lectura en la Escuela de Posgrado de la Universidad Católica Sedes Sapientiae (UCSS). 
"Primera mesa redonda sobre literatura peruana y sociología", el "Primer encuentro de narradores peruanos" y la "Mesa redonda sobre Todas las sangres". Por lo tanto, con base a un análisis comparativo entre los argumentos de Alberto Escobar, José Miguel Oviedo, Winston Orillo, Aníbal Portocarrero con Julio Ramón Ribeyro, se demostrará los puntos de conexión entre ellos. En ese sentido, se podrá demostrar el rol de crítico literario de Ribeyro expuesto en los artículos y ensayos de La caza sutil.

Palabras claves: Julio Ramón Ribeyro, crítica literaria, artículos, ensayos, debates literarios en el Perú.

\title{
JULIO RAMÓN RIBEYRO AND THE LITERARY DEBATES OF THE 1960s IN PERU
}

\begin{abstract}
The objective of our research is to show that the arguments developed by Julio Ramón Ribeyro around the novel, the representation of reality and literary techniques exposed in the articles and essays of La causa sutil (1976) coincide with the literary critics of their context. They manifest their arguments in the literary events that took place in 1965: the "First round table on Peruvian literature and sociology", the "First meeting of Peruvian storytellers" and the "Round table on Todas las sangres". Therefore, based on a comparative analysis between the arguments of Alberto Escobar, José Miguel Oviedo, Winston Orillo, Aníbal Portocarrero and Julio Ramón Ribeyro, the points of connection between them will be demonstrated. In that sense, it will be possible to demonstrate Ribeyro's role as literary critic exposed in the articles and essays of La caza sutil.
\end{abstract}

Keywords: Julio Ramón Ribeyro, literary criticism, articles, essays, literary debates in Peru.

\section{Introducción}

Cl libro La caza sutil (1976), de Julio Ramón Ribeyro, contiene diversos artículos y Censayos sobre crítica literaria. Todos ellos aparecieron antes en diversos medios entre los años 1953 y 1975. A partir de sus textos ensayísticos se puede identificar otra faceta de Ribeyro en torno a la literatura: la crítica literaria. Cabe destacar que, uno de sus primeros textos literarios fue "En torno a los diarios íntimos", publicado en 1953. Se debe recordar también que Ribeyro publicó "La vida gris" en 1949, "La huella", "El cuarto sin enumerar" y "La careta" en 1952. Estos fueron sus primeros cuentos antes de la aparición de Los gallinazos sin plumas en 1955. En ese sentido, se demuestra que los primeros textos literarios de Ribeyro giran en torno al cuento y ensayo o artículo. 
Las ediciones que tiene La caza sutil son una muestra del desinterés por parte de la crítica literaria. Si bien existen pocos estudios sobre el género ensayístico en el Perú, esto se apoya en las pocas reediciones que han tenido las obras de ensayos o artículos de escritores peruanos. Ante esto, la intención de publicar todos los ensayos y artículos de Ribeyro se ha logrado con la última edición de 2016. Esta edición contempla la compilación de todos sus textos. Sin embargo, se debe mencionar El archivo personal de Julio Ramón Ribeyro (2006) de Luis Fuentes ${ }^{1}$ y la edición de la Universidad Diego Portales titulada La caza sutil y otros textos. Un desaprensivo paseo entre libros y autores (2012). Estas fueron las ediciones que intentaron recopilar todos tus textos ensayísticos. ${ }^{2}$

No solo mediante los ensayos o artículos de Ribeyro se evidencia su faceta de crítico literario. Las entrevistas brindadas y recopiladas por Jorge Coaguila en Ribeyro, la palabra inmortal (2008) y Las respuestas del mudo (2011) son un testimonio importante de las ideas y argumentos que complementan los textos en La caza sutil. A pesar de ello, aún es escasa la recepción crítica y existe poco interés por la prosa de no ficción de Ribeyro. En ese sentido, es necesaria el estudio de la obra ensayística de Ribeyro y, en general, del género en el Perú.

Los diversos ensayos y artículos de Ribeyro abarcan diversos temas en torno a la literatura peruana y latinoamericana, literatura francesa, entre otros. De manera específica, aborda tres temas que son debatidos y discutidos por la crítica literaria y escritores de su contexto: la novela, la representación de la realidad y las técnicas literarias. Estos temas se desarrollaron en dos mesas redondas y un encuentro literario de suma importancia: la denominada "Primera mesa redonda sobre literatura peruana y sociología" (1965), el "Primer encuentro de narradores peruanos" (1965) y la "Mesa redonda sobre Todas las sangres" $(1965)^{3}$. En estos eventos participaron escritores de la literatura peruana como Enrique

$1 \quad$ Luis Fuentes menciona la complicada tarea de entender el mundo bibliográfico de Ribeyro: "Ediciones agotadas, librerías mal surtidas, ediciones no reeditadas, bibliotecas incompletas, y bibliografías con muchos errores determinaron que esta inquietud se convirtiera en una necesidad por aclarar el panorama bibliográfico de Julio Ramón” (2006, p. 28).

2 El crítico y biógrafo Jorge Coaguila menciona:

¿Por qué no escribió mucho más artículos en esos más de cuatro decenios? Es evidente que tenía las puertas abiertas en los más importantes diarios limeños. La parquedad del número de artículos se debe a que no buscaba escribir sobre la actualidad, de la que -como el protagonista de sus Dichos de Luder (1989) - se sentía cada vez más ajeno. Escribía cuando consideraba que tenía algo importante que decir. (2008, p. 122)

3 Todas las sangres (1964) es la novela de José María Arguedas que fue debatida y discutida sobre diversos temas en una mesa redonda con la participación de críticos literarios y escritores peruanos. Dorian Espezúa realiza un estudio de cada uno de los argumentos vertidos en este acontecimiento y los confronta con los 
Solari Swayne, José María Arguedas, Ciro Alegría, Oswaldo Reynoso, Sebastián Salazar Bondy, entre otros. Asimismo, se contó con la presencia de críticos literarios, profesores y estudiosos de la literatura como Alberto Escobar ${ }^{4}$, José Miguel Oviedo, Winston Orrillo y Aníbal Portocarrero.

El objetivo de nuestra investigación es evidenciar que los argumentos desarrollados por Julio Ramón Ribeyro en torno a la novela, la representación de la realidad y técnicas literarias expuestos en los artículos y ensayos de La caza sutil coinciden con los críticos literarios que participaron en los debates literarios de los años 60 en el Perú. Por ello, será imprescindible destacar los argumentos de Alberto Escobar, José Miguel Oviedo, Winston Orrillo y Aníbal Portocarrero en torno a los temas que se debatieron y discutieron en los eventos de 1965. En ese sentido, se podrá demostrar el rol de crítico literario que ejerció Ribeyro en su libro La caza sutil.

\section{La novela para Julio Ramón Ribeyro y Alberto Escobar}

T a relación entre Julio Ramón Ribeyro y Alberto Escobar data de los años 1946 y 1952. Según Miguel Gutiérrez (2008), Alberto Escobar es uno de estudiosos de la literatura de la denominada Generación del 50. Esta generación no solo estuvo conformada por narradores y poetas; sino, también por artistas plásticos, filósofos e intelectuales que ayudaron a la formación cultural del país. De manera específica, Escobar se ha convertido en uno de los pilares de los estudios literarios en el Perú y nadie podrá dudar de esta labor. Por otra parte, Ribeyro y Escobar no tuvieron una mesa o encuentro de discusión o debate; sin embargo, compartían una línea argumental en torno a la novela, siendo más concreto, a la novela peruana. Esto se puede evidenciar mediante las declaraciones de Escobar en las

argumentos de Arguedas - partícipe de aquel evento- en su libro Todas las sangres en debate. Cientificos sociales versus criticos literarios (2011). El autor menciona:

Este libro es una crónica del (des)encuentro entre científicos sociales y críticos literarios que se produjo el 23 de junio de 1965 en el Instituto de Estudios Peruanos (IEP) para debatir Todas las sangres (TLS), la novela de José María Arguedas (1911 - 1969), publicada en 1964. (...) Puede considerarse una gran reseńa que produce un texto que lee otros textos por cuanto no plantea una interpretación, sino más bien una comprensión del debate. En efecto, no hay aquí una pretendida superioridad de la interpretación frente a la explicación y comprensión. Lo que fundamentalmente hago es «dejar hablar» a los participantes en el debate y a los que opinaron sobre la novela y sobre el debate que ella originó. (p. 11)

4 Carlos García-Bedoya realiza un idóneo artículo sobre Alberto Escobar destacando su semblanza y tiene "la difícil pero honrosa tarea de examinar el aporte del crítico por antonomasia de la generación” (2006, p. 235) 
mesas redondas y encuentro que se suscitaron en el año 1965. De tal manera, las opiniones y argumentos de Escobar tendrán puntos de coincidencia con los argumentos en los artículos y ensayos de La caza sutil de Julio Ramón Ribeyro.

Alberto Escobar sostiene, entre los diálogos con otros escritores, dos líneas argumentales sobre la novela. La primera se menciona en la "Primera mesa redonda sobre literatura peruana y sociología”. Esta permite brindar un acercamiento idóneo al análisis de la novela. En ese sentido, Escobar menciona lo esencial para el crítico literario en su accionar analítico de este género literario:

No se puede negar que en la novela, más que en otros géneros literarios se precisa muy bien que la construcción literaria, que el trabajo del escritor se nutre de una forma de conocimientos, y que la novela es, en sí, una manera de conocer la realidad, al construirla, al modificarla, al transformarla, al recrearla, es siempre una manera de conocimientos. Ahora, la divergencia con el trabajo del estudioso de las ciencias sociales reside en que no hay duda que la novela como toda obra de arte es una forma de conocimiento, predominantemente intuitivo (...) (Escobar, 2003, p. 41)

La premisa que se establece con base a los argumentos de Escobar es entender la novela como una construcción literaria que puede brindar conocimientos en torno a la realidad la cual ha sido construida. Estos se brindan por medio de intuiciones por parte del lector -sociólogo o crítico literario- debido a que se trata de una obra artística. En otras palabras, Escobar sostiene que se puede aprender, tanto racional como intuitivamente, de la realidad por medio de una novela. Por otra parte, esta podrá transgredir, recrear o reconstruir la realidad aludida.

Por otro lado, Escobar manifiesta, con mayor precisión, la importancia de la construcción literaria en la novela. Esta exige al crítico literario para que pueda desmenuzarla y analizar cada parte de su estructura. Este argumento ayuda a entender el interés por analizar la obra literaria de manera objetiva por parte de Alberto Escobar. Inclusive, será clave para los estudios literarios en el Perú: 
Para avanzar un punto más me toca a mí decir en algo lo que yo como lector y como estudioso de la novela encuentro en la obra de Arguedas. Y partiré por una premisa, por un elemento previo. En la crítica literaria, el sentido del mensaje, como un desprendimiento simbólico, como un metalenguaje que emana de la obra literaria, está dado para el crítico no por la suma de los valores semánticos, de los valores del contenido, sino por un proceso de sublimación o de decantación. (Escobar, 2000, p. 21)

Este argumento es mencionado en la "Mesa redonda sobre Todas las sangres", organizada por el Instituto de Estudios Peruanos (IEP). En ella participación José María Arguedas, José Miguel Oviedo, Henri Favre, entre otros. Escobar gira su comentario en torno a la novela de Arguedas y menciona la importancia de la construcción literaria dentro de esta. Asimismo, centra su atención al abordaje del crítico de analizar tanto cada parte de la obra como su totalidad. Esto permite entender el argumento de Escobar con respecto a la construcción literaria de una novela y cómo debe proceder el crítico literario ante el análisis y observación de la misma. En otras palabras, las dos líneas argumentales de Escobar se sostienen en el aprendizaje de la realidad aludida que se logra por medio de una novela con una construcción literaria estructurada y el rol del crítico en torno al análisis de esta construcción para una mayor comprensión de la realidad mencionada. Estos argumentos coinciden con las ideas expresadas en artículos y ensayos de Ribeyro.

En 1958, Ribeyro publicó un artículo denominado "Sobre La tierra prometida (Crítica literaria y novela)". Este tuvo como intención ser parte del debate de diversos artículos que giraron en torno a la novela de Luis Felipe Angell. Entre ellos estuvieron José María Arguedas, Luis Jaime Cisneros, Mario Castro Arenas y Sebastián Salazar Bondy. El artículo refleja un argumento claro en torno al rol del crítico literario desde la perspectiva de Ribeyro. Si bien ahonda en el análisis de la novela de Angell, nos presenta una clara idea sobre la crítica literaria:

Lo que es de lamentar es que los artículos de los autores citados no se ocupen, en rigor, de crítica literaria. Todos, sin excepción, soslayan el problema. Ciertamente, los temas debatidos son del más alto interés teórico. Es muy ilustrativo discutir sobre la verdad o falsedad de las peripecias narradas por Angell o polemizar sobre el derecho que asiste al artista para suplir con la 
imaginación los datos que no suministra su experiencia. Pero creemos que todas estas consideraciones pertenecen algunas a la etnología, otras a los capítulos más abstractos de la ciencia literaria. Ellas tienen poco o nada que ver con la novela de Angell. No nos interesa saber si La tierra prometida es verdadera o falsa. Lo que nos interesa es saber si se trata de una buena o mala novela. (Ribeyro, 2012, p. 72)

Ribeyro realiza una crítica a la manera cómo se está abordando la novela La tierra prometida. Esto le sirve para mencionar que no existe crítica literaria. Además, señala que los participantes al intercambio de ideas evitan el problema central de juzgar la novela, centrándose en otro tema. De tal manera, Ribeyro argumenta que el interés en la novela debe recaer en un juicio valorativo. Sin embargo, esto se logra mediante el análisis de cada una de sus partes. Por ello, el artículo lo dedica a explicar varios aspectos de la novela y juzgarla en torno a estos. En ese sentido, Ribeyro coincide con Alberto Escobar en torno a un análisis de cada una de las partes de una construcción literaria, en este caso la novela $L a$ tierra prometida. Este trabajo debe ejercerlo el crítico literario.

En 1969, se publica el ensayo "Problemas del novelista actual". Ribeyro delimita los problemas a tres: el problema de la complejidad del mundo, el problema de la representación de la simultaneidad y el problema de la expropiación del territorio novelístico por otras disciplinas. Este último problema empieza desarrollando la manera como los novelistas han trasmitido conocimientos por medio de su obra de arte. No obstante, no se han logrado comparar con los estudios o investigaciones de los científicos. De tal manera, Ribeyro manifiesta que el objetivo de una novela no es transmitir conocimientos sino vivencias:

Toda esta argumentación sin embargo y felizmente es falsa. Falsa por una razón muy sencilla: porque en última instancia la función del novelista no es transmitir un "saber" sino una "vivencia”. La novela se dirige más a la afectividad que a la inteligencia. Novelistas y científicos pueden tratar los mismos temas, pero no con la misma intención ni con el mismo procedimiento. (Ribeyro, 2012, pp. $88-89)$ 
El argumento de Ribeyro brinda el objetivo de una novela en el lector. Con base que la novela sea expropiada por otra disciplina, en este caso las ciencias sociales o científicas, Ribeyro declara el elemento diferenciador de la novela y los novelistas. No obstante, se debe mencionar que antes de declarar el argumento de Ribeyro, este nombra cómo la novela se ha ido utilizando como fuente de conocimiento pero las investigaciones han refutados sus propuestas. De tal manera, Ribeyro reconoce que la novela sí ha sido utilizada como una fuente de conocimientos pero no está de acuerdo que su finalidad sea tal. Por el contrario, su argumento se inclina por una novela que transmite vivencias y estas sirvan como una fuente de conocimiento intuitivo, a diferencia de estudios científicos o sociales. En ese sentido, Ribeyro coincide con Escobar porque los dos argumentan que la novela posee y transmite conocimientos, priorizando la intuición por encima de lo racional. Por lo tanto, Ribeyro coincide con uno de los mejores críticos y estudios de la literatura peruana y fundador de los estudios literarios en el Perú. Esto no es mera casualidad debido a la cercanía y buena amistad entre ellos.

\section{Ribeyro, Escobar, Oviedo y Orillo sobre la representación de la realidad}

U Tno de los temas, que más debate y discusión ha dejado, gira en torno a la representación de la realidad por parte de los novelistas. En 1965, las dos mesas redondas y el encuentro de narradores se logran distinguir diversas ideas y argumentos sobre el tema. Julio Ramón Ribeyro no fue incluido dentro de estos eventos, sin embargo, desde su propia ventana, sea artículo o ensayo literario, logra manifestar sus argumentos sobre esta problemática. En esta oportunidad, Ribeyro no solo coincide con Alberto Escobar; sino, también con un joven José Miguel Oviedo y Winston Orillo. Estas personalidades participaron de los eventos mencionados y se establece una relación con los argumentos de artículos y ensayos de Ribeyro.

Los argumentos de Alberto Escobar en torno a la representación de la realidad por parte de los novelistas se sostienen en algunas declaraciones que emite dentro de la primera mesa redonda y el encuentro de narradores peruanos. Aquella es mencionada debido a que el debate permite a Escobar expresar su opinión sobre la relación entre la realidad y la obra literaria: 
Entonces, ante este nuevo planteamiento, para mí qué es lo que pasa, cuál es la relación que existe entre la obra literaria y la realidad. Yo creo y yo pregono que la obra literaria es una realidad sacada de la realidad, autónoma, independiente y que cuando hay que estudiarla desde el punto de vista literario, hay que estudiarla así, que sea el ingrediente básico, el argumento básico. (Escobar, 2003, pp. $43-44)$

Escobar manifiesta dos aspectos en su intervención. El primero se centra en colocar a la realidad como base para construcción de una novela. Esta nueva realidad, la novela, es una construcción literaria la cual debe ser juzgada por el crítico literario. El segundo es entender como principio básico que el crítico literario debe analizar esta construcción literaria o realidad autónoma, independiente de la realidad que sirve como base. En otras palabras, Escobar argumenta que la obra literaria es una construcción con base en la realidad. Toda obra literaria no surge de la imaginación absoluta, sino de una realidad objetiva. Además, el rol del crítico literario es analizar la construcción literaria más no la realidad objetiva. Por otra parte, Escobar agrega lo siguiente: "Entonces, toda obra literaria reproduce la realidad más que en sus detalles, en ciertas leyes ocultas y secretas, invisibles incluso para el hombre común cuando mira la realidad en que vive.” (Escobar, 2012, p. 44). De esta manera, se agrega en el argumento de Escobar que la novela u obra literaria reproduce la realidad y manifiesta aspectos no tan visibles. En ese sentido, el argumento clave de Escobar sobre la representación de la realidad por parte de los novelistas es una reproducción de la realidad en la novela visibilizando algunos elementos de aquella no tan claro de apreciar por el hombre. Por otra parte, el crítico Escobar señala que el rol de crítica literaria debe ser desentrañar estos nexos entre la realidad y la obra literaria, observar cuáles son sus conexiones y en qué medida se aborda esta relación.

Ahora bien, con respecto a José Miguel Oviedo, es necesario señalar que al día de hoy es reconocido como un crítico literario de trayectoria nacional e internacional. Entre sus obras destacan Mario Vargas Llosa: la invención de una realidad (1970), Breve historia del ensayo hispanoamericano (1991) y los cuatro volúmenes de la Historia de la literatura hispanoamericana (1995 - 2000). No obstante, en 1965 aún no era acreedor de tan vasta obra pero destacaban sus apreciaciones y opiniones en torno a la literatura. El tema de la representación de la realidad por parte de los novelistas fue parte de una intervención en la primera mesa redonda: 
La creación literaria, el arte, es el fruto de un choque entre las obsesiones personales y las experiencias que comparten el autor con todos sus contemporáneos. Ese choque produce una fusión de lo que siente y lo que piensa, que pueden ser como el día y la noche. Frente a esto, el creador adopta una actitud muy particular: aunque comparte con el resto una misma realidad, ella no le basta e inventa la suya. (...) Pero hay un momento en la cual esta realidad creada por el escritor aspira a confundirse con la realidad social. Tiene una intencionalidad cuyo alcance va más allá del mundo interior del escritor y amenaza con derribar los límites de la misma realidad, de transfigurarla o transgredirla. Una obra literaria no es un simple reflejo de la realidad, sino una interpretación de ella, a través de ciertas formas y técnicas. (Oviedo, 2003, p. 34)

Oviedo se refiere que el arte es un resultado de dos mundos: interno y externo del creador. Es decir, las vivencias personales y externas de nuestro creador dan como resultado una obra literaria. Asimismo, esta obra está enfocada desde un punto de vista. El creador tiene una perspectiva de la realidad que lo rodea que, posteriormente, es plasmada en una obra literaria, agregando ciertos elementos. Seguidamente, Oviedo manifiesta que la obra literaria es otra realidad que, de alguna manera, reinventa la realidad social, incluso la interpela o la transforma. En otras palabras, Oviedo sostiene el argumento que la obra literaria posee una realidad al margen de la realidad de la cual es construida. En ese sentido, se evidencia que la representación de la realidad por parte de los novelistas, desde la perspectiva de Oviedo, no solo es un reflejo sino una interpretación que se configura en torno al resultado de los dos mundos. Por otra parte, Oviedo en su participación del "Primer encuentro de narradores peruanos" reafirma su posición en torno a este tema. Incluso, sintetiza sus opiniones:

Yo pienso que todo el que escribe una novela está en contra, en contra de algo puesto que escribe; y esta posición contraria, ese voto en contra está en su novela, en la representación de la realidad. Hay dos cosas, pues, la realidad previa, la realidad experimental, digamos, experimentada, y la realidad representacional, la realidad mentirosa que surge del arte. Yo no creo que la 
vida rica, variada o auténtica de un escritor garantice la riqueza, la perfección, la autenticidad de la realidad que él representará en su novela. (Oviedo, 1986, p. 114)

En esta intervención, Oviedo logra precisar su argumento sobre la representación de la realidad. Esta se le añade a la novela y menciona la conjunción de las dos realidades que un escritor posee con base a la construcción literaria. Sin embargo, el mayor aporte de esta intervención se debe al impacto de la novela dentro de la realidad social. Este impacto se evidencia mediante el uso de la novela como herramienta de contraste. En ese sentido, el argumento de Oviedo responde a una participación transgresora de la novela en la realidad social.

Con respecto a Winston Orillo, podemos decir que fue profesor de literatura en la Universidad de Lambayeque. Posee un doctorado por la Universidad Nacional Mayor de San Marcos y ha destacado como poeta. En relación al "Primer encuentro de narradores peruanos" participó en calidad de catedrático. Por ello, sus intervenciones son relevantes, debido a la propuesta de esta investigación. Sin embargo, en comparación con los demás participantes, sus intervenciones son mínimas pero rescataremos dos que giran en torno al tema de la representación de la realidad. Orillo empieza su intervención considerando que las realidades representadas en las novelas de Arguedas y Alegría no son realidades cercanas a él ni a sus contemporáneos. En comparación con las realidades de Oswaldo Reynoso que sí lograba cierta identificación. Esto lo lleva a declarar lo siguiente. "Hay que plantearse este problema en los términos de la realidad para el novelista y la realidad para el lector." (Orillo, 1986, p. 116). Su intervención permite entender un aspecto no muy abordado por los críticos y escritores en torno a este encuentro: la realidad del lector.

El argumento de Orillo se evidencia en establecer realidades distintas de cada uno de los escritores. Por ejemplo, realiza una distinción entre Arguedas, Alegría y Reynoso. Esto responde a una perspectiva de cada autor en representar una realidad en torno a sus experiencias o vivencias personales. Por ello, se comprende que Orillo sienta mayor afinidad con las obras de Reynoso sobre las obras de los autores indigenistas. En ese sentido, el argumento de Orillo se centra en establecer una representación de la realidad por cada perspectiva de un autor o novelista. Por otro lado, Orillo en otra intervención con intención de responder a Reynoso alude al rol del escritor con la realidad que lo rodea: 
Muy brevemente quiero referirme a una afirmación de Oswaldo Reynoso, de hace unos minutos, antes de que se levantara la sesión. Él dice que la realidad es caótica y que el escritor tiene que ordenar esa realidad en una realidad nueva; sucede que el problema es diferente, la realidad tiene su orden y simplemente la literatura tiene un orden diferente a esa realidad. (Orillo, 1986, p. 132)

El catedrático Orillo menciona una distinción entre la realidad que envuelve al novelista o creador y la realidad literaria. Esta es una "realidad nueva" con base en la realidad que, en este caso, es caótica. Además, la noción de brindarle un orden a esta realidad es un ejercicio que realiza el novelista o creador para una representación de una realidad objetiva. En ese sentido, el argumento clave de Orillo en torno al tema se refiere a la clara distinción entre una realidad como base de la realidad plasmada en la obra literaria.

En 1969, Ribeyro publica el artículo "Del espejo de Stendhal al espejo de Proust". Este contiene las perspectivas de los autores franceses al momento de representar la realidad en sus novelas. Ribeyro describe las concepciones de espejo en Stendhal y Proust con la finalidad de realizar un panorama de la novela. También, se permite esbozar la manera cómo abordan la realidad en un periodo de un siglo. Seguidamente, logra sentar las perspectivas de los autores franceses en torno a la concepción de espejo y su producto en torno a las novelas publicadas, específicamente Rojo y negro y En busca del tiempo perdido. Sin embargo, Ribeyro proporciona un argumento contundente en torno a la representación de la realidad y cómo esta interfiere en ella:

Es importante destacar la noción de prisma -implícitamente contenida en la definición proustiana-, porque ella es uno de los elementos esenciales de la novela moderna, impensable un siglo antes. El principio stendhaliano de la novela-espejo, que tanta fortuna tuvo en el siglo XIX, es sustituido por Proust por el de autor-volumen refractante. Esta sustitución supone un grado más de conciencia del quehacer novelesco. En los autores más importantes de nuestro siglo encontramos esa visión prismática de la realidad, se ponga el acento sobre la deformación de su imagen, la descomposición de sus elementos o la multiplicación de sus perspectivas. La noción de prisma permite además 
superar la dicotomía invención-reproducción, origen de debates tan largos como estériles, y reemplazarla por la noción de transformación. El novelista no se limita a jugar con los elementos imaginarios o a reproducir elementos reales, sino que se sirve de ambos para fundirlos en una entidad diferente, la entidad literaria, mundo paralelo al nuestro que lo resume, lo ordena, lo corrige, lo interpreta, lo comenta, lo explica, lo enriquece y, en ciertos casos, lo suplanta. (Ribeyro, 2012, p. 137)

Los argumentos de Ribeyro se sostienen en dos concepciones que desarrolla en torno a Stendhal y Proust. Esto le sirve para esclarecer los aspectos relevantes de la novela moderna. La noción de prisma es acertada con base a las nociones de espejo de los autores franceses. Sin embargo, Ribeyro brinda su argumento en torno a la representación de la realidad por parte de los novelistas. En primer lugar, se refiere a descartar la dicotomía de invención-reproducción y elegir el concepto de transformación. De esta manera, no niega que la novela representa la realidad -aspecto que coincide con Escobar, Oviedo y Orillosino también que aquella transforma la realidad que le sirve de sustento. En segundo lugar, Ribeyro se refiere a dos realidades que se conjugan en la novela. En este caso, los elementos reales e imaginarios que posee el novelista los reúnen en la obra y esta es una nueva realidad, diferente y autónoma. Por ello, coincide con sus contemporáneos en torno a la realidad como fundamento básico para la construcción literaria. Esto es posible por medio de estos dos mundos o realidades que posee el creador o novelista. Por último, Ribeyro menciona la intencionalidad de la novela u obra literaria en la sociedad. Esta logra criticar, modificar u reordenar la realidad. Es decir, la novela no solo representa o transforma, al mismo tiempo, interfiere e interviene en la realidad que le ha servido como fundamento básico. Este argumento va en paralelo solo con José Miguel Oviedo. En ese sentido, los argumentos de Ribeyro en torno a la representación de la realidad por parte de los novelistas coincide y va en paralelo con Alberto Escobar y José Miguel Oviedo, críticos literarios, y Winston Orillo, catedrático de literatura.

\section{Técnicas literarias}

os eventos llevados a cabo en 1965 abordan el tema de las técnicas literarias con mayor
atención a la opinión de los escritores. Probablemente, el centro de atención fue Carlos 
Eduardo Zavaleta en el "Primer encuentro de narradores peruanos". Sin embargo, críticos literarios y un profesor de literatura logran intervenir en estos temas. Es imprescindible abordar esta temática debido a la relación que se establece con la novela y la representación de la realidad por parte de los novelistas. Asimismo, la oportunidad de escuchar a Ciro Alegría y José María Arguedas en comparación con los escritores de la Generación de 50 es evidenciar un proceso evolutivo en la narración peruana. Esta atraviesa las obras de Julio Ramón Ribeyro. En ese sentido, es importante presentar un panorama sobre los argumentos de Ribeyro y las coincidencias que se establece con Alberto Escobar, José Miguel Oviedo y Aníbal Portocarrero.

En el "Primer encuentro de narradores peruanos" se establecen tres temáticas para los tres debates en cuestión. El primero trata sobre el novelista y la realidad. El segundo concierne al sentido y valor de las técnicas literarias. Por último, se debate sobre la evaluación del proceso de la novela peruana. Escobar participa de los tres temas de manera fructífera. En el caso de las técnicas literarias, Escobar menciona:

Yo no creo que se pueden hacer postulaciones sobre la técnica y sobre la intuición de manera general; yo no creo que se pueda decir, como ha dicho Sebastián, que es necesario ver qué técnicas han utilizado los autores peruanos y ver qué técnicas nos falta emplear. Eso me parece falso, porque creo que la técnica está condicionada por la motivación, por la experiencia concreta que significa la realización de la obra literaria y no se puede prescribir qué técnicas se van a usar. El conocimiento de la técnica se ha obtenido por estudio, por inferencia y forma parte de una tradición; pero la elección de una palabra o de un recurso expositivo o de un efecto final o de una forma estrófica, escribir un verso libre o en soneto, todo esto está motivado por una relación oscura entre el creador y lo que quiere decir. (Escobar, 1986, p. 199)

Escobar menciona la importancia de la técnica literaria dentro de la construcción de una obra. La técnica literaria no debe prevalecer ante el tema o motivo de una obra. Por el contrario, el motivo debe ser primordial dentro de la elaboración de una obra literaria y la técnica solo debe estar subordinada. También, Escobar agrega que la técnica de los escritores son parte de una trayectoria, de una tradición literaria. En ese sentido, el argumento de 
Escobar se sostiene porque considera la técnica un recurso instrumental, más no un recurso imprescindible en la elaboración de una obra literaria. Si bien existen obras donde prevalece el aspecto técnico, debe ser primordial tener en cuenta la perspectiva, el tema o motivo de lo que se quiere plasmar en la obra literaria.

José Miguel Oviedo en una intervención menciona brevemente el tema de las técnicas literarias, incluso lo aborda con otras alternativas que posee el escritor al momento de elaborar su obra literaria. Esto lo aclara para lograr diferenciar el texto literario del documento social. De tal manera. Oviedo presenta su argumento en torno al tema:

$\mathrm{Al}$ parecer hemos estado omitiendo un elemento importante al examinar la literatura como documento social. Hemos estado hablando de lo social más bien como temática o materia, es decir, el plano semántico.

Pero estamos olvidando el otro aspecto del lenguaje narrativo, el aspecto puramente formal, que es esencial en el fenómeno literario. Creo que para el sociólogo es muy importante entender cómo y por qué el escritor usa, por ejemplo, el habla popular, las formas a veces mestizadas, propias de una cultura plural como la del Perú. (Oviedo, 2003, p. 50)

Oviedo no realiza una diferencia entre la técnica y la temática en la elaboración de una obra literaria; sin embargo, sí le da la atención necesaria al abordar este tipo de temas. Oviedo argumenta que la técnica o lo formal debe ser importante para un estudio sociológico. Es decir, se manifiesta la intención de buscar alguna respuesta de la elaboración del documento literario en la técnica literaria. No obstante, no solo menciona que se debe tomar en cuenta; sino, también, complementar con otras alternativas que servirán para construir la obra literaria, por ejemplo, la lengua, el lenguaje, etc. En ese sentido, el argumento de Oviedo se justifica en brindarle importancia a la técnica literaria o el aspecto formal para lograr entender y dar una diferencia entre un documento social y un texto literario.

Para el caso de Aníbal Portocarrero, podemos mencionar que fue catedrático de la Universidad Nacional San Agustín en Arequipa. Si bien su obra trascendió en el género poético, estuvo como invitado en el "Primer encuentro de narradores peruanos" como profesor de literatura. Su poca participación en los debates no permite entender a cabalidad 
su postura frente a las técnicas literarias y otras alternativas que utiliza el creador para la construcción de la obra literaria. Portocarrero en su intervención tiene como intención apoyar la postura de Arguedas, puesto que, se siente un gran admirador del autor de $E l$ Sexto. Ante esto, Portocarrero argumenta su posición con base en Saussure:

Cuando Saussure nos habla del circuito del habla (que ha sido bastante criticado, por supuesto, pero en el fondo subsiste todavía incluso en las críticas de Bühler), él nos hace ver que este circuito se da solamente entre dos elementos fundamentales: el elemento hablante y el elemento oyente, y cierra el circuito entre estos dos elementos. La crítica que ha hecho Karl Bühler, yo la he leído en el texto de Lenguaje de Zubizarreta, fue precisamente por el hecho que Saussure había prescindido de un tercer elemento fundamental interviniente en el circuito, que es la realidad, o como él la llama, la fuente de estímulo. (Portocarrero, 1986, pp. 148 - 149)

Del argumento de Portocarrero se entiende que la participación de los elementos, mencionados por la propuesta de Saussure, forma parte de una realidad que es aludida. Sin embargo, es interesante entender que Portocarrero no se refiere a técnicas literarias; sino, más bien, al lenguaje que se debe utilizar en la construcción literaria. Por tal motivo, su intervención es clave porque permite brindar ciertos conocimientos en torno a Saussure y el aporte que brinda dentro del tema. En ese sentido, Portocarrero solo argumenta una idea. Esta se relaciona con el tercer elemento que Saussure no agrega. La fuente de estímulos vendría a ser aquella realidad que después es aludida por medio del lenguaje en una obra literaria. Por ello, su argumento se centra en el uso de una lengua apropiada para la elaboración de la obra literaria.

En 1973, Ribeyro publicó el ensayo "Las alternativas del novelista”. En el desarrollo de su ensayo enumera diversas alternativas que el novelista tiene al momento de elaborar la novela. Sin embargo, prestaremos atención a dos que coinciden con los argumentos de Escobar, Oviedo y Portocarrero. La primera alternativa del novelista, mencionada por Ribeyro, es la lengua: 
La primera alternativa del novelista es la de la lengua: en qué lengua va a escribir. Esta alternativa puede parecer quizá algo rara, pero es más frecuente de lo que parece. En el caso del Perú, que es un país bilingüe, es una alternativa que se ha presentado y que se presentará -incluso en el futuro- si la enseñanza del quechua, como está previsto, se va intensificando. (Ribeyro, 2012, p. 109)

Si Ribeyro menciona a la lengua como una de las primeras alternativas del novelista es porque considera que será clave en el futuro la construcción literaria de este. Particularmente, en el Perú, se reconoce el quechua como otra lengua y existen pocos autores que escriben sus obras en quechua, a pesar que se ha intensificado. No debemos dejar de mencionar que es determinante la elección de la lengua al momento de iniciar una obra literaria. Un claro ejemplo de elección de la lengua es el caso de los primeros textos de Arguedas. En ese sentido, el argumento de Ribeyro tiene un punto de conexión con el argumento de Portocarrero. Puesto que, los dos postulan que la utilidad de una lengua es imprescindible en la construcción de una obra literaria. Por otra parte, Ribeyro se refiere a la técnica literaria como otra alternativa del novelista en "Las alternativas del novelista":

La técnica no es otra cosa que la manera de organizar y ejecutar un relato, de modo que éste al alcance su máxima de eficacia. La técnica siempre ha preocupado a los novelistas pero en los últimos años esta preocupación se ha exacerbado, hasta el punto de que ha pasado muchas veces a convertirse en un fin en sí misma y no en un instrumento al servicio de algo que la trascienda. (Ribeyro, 2012, p. 115)

Ribeyro menciona la importancia de la técnica dentro de la elaboración de una novela. Esta logra cierto orden, jerarquía y ejecución dentro de la construcción literaria, Cabe mencionar que dichas acciones son parte de las decisiones del autor o novelista. Asimismo, manifiesta que la técnica no debe ser utilizada como plantilla en relación a la temática que se intenta transmitir. Por el contrario, solo debe ser un instrumento adicional para canalizar la idea o noción que debe superarla. Esto coincide con Escobar porque la técnica solo debe ser un instrumento dentro de la elaboración de la novela y mantiene una relación estrecha con Oviedo, debido a que el crítico literario, sustenta y argumenta que para conocer qué tipo de 
documentos es la novela es necesario saber cómo se ordenó, organizó o qué estructura tiene la novela. En ese sentido, los argumentos de Ribeyro mantienen un mismo camino que los argumentos de Escobar y Oviedo en torno a la técnica literarias.

\section{Conclusiones}

Tarios de los artículos y ensayos contenidos en La caza sutil de Julio Ramón Ribeyro coinciden con críticos literarios (Alberto Escobar y José Miguel Oviedo) y profesores de la literatura (Winston Orillo y Aníbal Portocarrero). Estos se limitan a tres temas que son la novela, la representación de la realidad y las técnicas literarias.

La primera conclusión responde a la problemática de la novela. Ribeyro coincide con el crítico literario Alberto Escobar. Sus argumentos se relacionan en torno al conocimiento intuitivo que posee y transmite la novela. Es decir, toda novela posee una construcción literaria que contiene conocimientos, predominando la intuición por encima de lo racional.

La segunda conclusión concierne al tema de la representación de la realidad por parte de los novelistas. El argumento de Ribeyro concierne en comprender las dos realidades: una, literaria; otra, realidad real. Esta es el sustento básico de la nueva realidad o "entidad literaria". Escobar, Oviedo y Orillo coinciden con Ribeyro en torno a este tema de distinguir estas dos realidades o mundos y que uno de ellos es el sustento básico del otro. Además, mencionan que la labor del crítico literario es desentrañar los puntos de conexión y las relaciones entre estas dos entidades. En el caso de Escobar, coincide que la obra literaria es un reproducción de la realidad añadiendo algunos elementos; Oviedo considera que la novela u obra literaria no solo es una representación de la realidad; sino, también una interpretación de esta; Orillo argumenta que existe dos realidades: la primera con respecto a la que envuelve al escritor y la segunda que es construida con base a esta. En ese sentido, se justifica que los argumentos de Ribeyro y los argumentos de Escobar, Oviedo y Orillo coinciden con respecto al tema de la representación de la realidad por parte de los novelistas.

La tercera y última conclusión se centra en las técnicas literarias y otras alternativas. Ribeyro coincide con Escobar, Oviedo y Portocarrero en diversos aspectos. Escobar menciona que la técnica tiene un fin instrumental que debe ser operada en torno al tema elegido, es decir, el motivo o tema prevalece a la técnica literaria; Oviedo manifiesta que la técnica podrá indicarnos si el documento es social o literario, pero la utilidad de la técnica nos ayuda a comprender cómo interviene el novelista en la obra literaria; Portocarrero menciona un aspecto no muy abordado por los críticos, la lengua. Este aspecto coincide 
con Ribeyro debido a que es parte de una de las alternativas que el novelista debe elegir para la construcción de la obra literaria. Ribeyro comparte los argumentos de los críticos literarios y el catedrático de literatura. Estos permiten desentrañar temas que están ligados a la crítica literaria.

Para finalizar, se logra demostrar que los argumentos en los artículos y ensayos que más tarde conformarían La caza sutil de Julio Ramón Ribeyro coinciden con los argumentos de los críticos literarios (Escobar, Oviedo) y profesores y estudiosos de la literatura (Orillo, Portocarrero). En ese sentido, no es arriesgado mencionar que Ribeyro asume un rol de crítico literario en sus artículos y ensayos. Sin embargo, aún queda por desentrañar las coincidencias que mantiene con los narradores de su generación pero este tema merecería ser abordado de forma más amplia en un nuevo artículo.

\section{Referencias}

Alegría, C.; Arguedas, J.; Hernández, A.; Izquierdo, F.; Meneses, P.; Reynoso, O.; Silva, O.; Salazar, S.; Vargas, E. \& Zavaleta, C. (1986). Primer encuentro de narradores peruanos (2. a ed.). Lima, Perú: Latinoamericana Editores.

Coaguila, J. (2008). Ribeyro, la palabra inmortal (3. ${ }^{\text {a }}$ ed.). Iquitos, Perú: Tierra Nueva Editores.

Espezúa, D. (2011). Todas las sangres en debate. Cientificos sociales versus criticos literarios. Lima, Perú: Magreb.

Fuentes, L. (2006). El archivo personal de Julio Ramón Ribeyro. Lima, Perú: Instituto Raúl Porras Barrenechea/Fondo Editorial de la Universidad Nacional Mayor de San Marcos.

García-Bedoya, C. (2006). Alberto Escobar y los estudios literarios en el Perú. San Marcos, $24,235-245$.

Gutiérrez, M. (2008). La generación del 50: un mundo dividido. Lima, Perú: Ediciones Sétimo Ensayo.

Pinilla, C. (Ed.). (2003). Primera mesa redonda sobre literatura peruana y sociología del 26 de mayo de 1965. Lima, Perú: Instituto de Estudios Peruanos.

Ribeyro, J. (2012). La caza sutil y otros textos. Un desaprensivo paseo entre libros y autores. 


\section{PEDRO CARLOS ESPINOZA HUAROTO}

Santiago de Chile, Chile: Universidad Diego Portales.

Rochabrún, G. (2000). La mesa redonda sobre Todas las sangres: 23 de junio de 1965. Lima, Perú: Instituto de Estudios Peruanos, Pontificia Universidad Católica del Perú. 